\title{
Visual search is not blind to emotion
}

\author{
Cory GerRitsen \\ York University, Toronto, Ontario, Canada \\ AleXandra Frischen \\ Macquarie Center for Cognitive Science, Sydney, Australia \\ AdAM BlaKe \\ York University, Toronto, Ontario, Canada \\ DANIEL SMiLeK \\ University of Waterloo, Waterloo, Ontario, Canada \\ AND \\ John D. EASTwood \\ York University, Toronto, Ontario, Canada
}

\begin{abstract}
A series of three visual search tasks revealed more efficient search for hostile than for peaceful faces among neutral face distractors. Given that this effect has been observed inconsistently in prior literature, meta-analytic methods were employed for evaluating data across three experiments in order to develop a more valid estimate of the potentially small effect size. Furthermore, in the present experiments, different emotional meanings were conditioned to identical faces across observers, thus eliminating confounds between the physical characteristics and the emotional valences of the face stimuli. On the basis of the present findings, we argue that the visual system is capable of determining a face's emotional valence before the face becomes the focus of attention, and that emotional valence can be used by the visual system to determine subsequent attention allocation. However, meta-analytic results indicate that emotional valence makes a relatively small contribution to search efficiency in the present search context.
\end{abstract}

Faces convey a wide range of valuable information to an observer. Ethologically, the emotional information conveyed by faces may be especially important because it may signal environmental threat. For example, an angry face might signal threat from an angry individual, or a fearful face may indicate a threat that another individual has detected. Given the meaningfulness of such information and the frequency with which it is encountered, one might expect a facial expression of emotion to be a valuable criterion for the allocation of attention (see, e.g., Hansen \& Hansen, 1988; Öhman, 1993).

Allocating attention to parts of a visual scene is necessary because our visual system cannot completely integrate, identify, or otherwise process every element of the environment each moment (see, e.g., Kahneman \& Treisman, 1984; Tsotsos, 1997). The visual system must select, in an ongoing fashion, the elements that will receive limited processing resources. This selection must be guided by information that is already available to the system prior to the allocation of focal attention (Wolfe, 2003). Given these assumptions, the present work seeks to address two questions: Can the emotional valence of faces be perceived prior to the allocation of focal attention (i.e., preattentively)? If so, does it influence the subsequent allocation of focal attention? The present investigation employs the visual search task in order to address these questions.

The visual search task is commonly used to investigate the allocation of spatial attention. In this task, a target item is placed among a varying number of distractors, and its presence, location, or identity must be reported; this allows one to assess the effects of increasing the number of distractor items in the display. If finding a particular target does not require focal attention, its presence will be reported with the same ease, regardless of the number of distractors surrounding it. Such a target is said to "pop out" of the display (i.e., to be processed without attention, in parallel with distractor items, or preattentively) (see, e.g., Treisman \& Gelade, 1980). However, searches for targets that do not possess this pop-out quality require additional time for each added distractor, because attention must be shifted among display items until the target is located. Thus, the slope of a function relating reaction time (RT) to the number of distractors is flat for items that pop

C. Gerritsen, cjgerrit@yorku.ca 
out of their respective distractor contexts and is steeper for items that do not.

Although the presence of pop-out is commonly used in the visual search literature as a touchstone for preattentive or parallel processing, its value as the sole indicator of such processing is questionable. Parallel or preattentive processes involved in search may be accompanied by serial or postattentive processes, and therefore may be present in searches that do not show pop-out. Many authors (e.g., Duncan \& Humphreys, 1989; Wolfe, Cave, \& Franzel, 1989; see Wolfe, 1998, for a review) have proposed alternatives to the all-or-none interpretation of visual search findings.

Wolfe (1998) critiqued the distinction between parallel and serial search on the basis of a review of nearly 1 million search trials that showed a unimodal distribution of search slopes, as opposed to the bimodal distribution that would be predicted by the all-or-none model. Brown, Huey, and Findlay (1997) observed saccadic eye movements during search and concluded that some search processes traditionally considered to be serial likely incorporate some parallel processes. As opposed to the dichotomous hypothesis of serial versus parallel processes, which dominated the early visual search literature, it may be more valid to examine varying degrees of "efficiency" of search (Wolfe, 1989). Efficiency is defined as the degree to which each additional distractor slows search for a target item.

In order to investigate the degrees of efficiency with which faces with different emotional expressions could be detected, Eastwood, Smilek, and Merikle (2001) compared the relative search performance for two emotionally valenced face targets. In their experiments, distractor contexts were held constant, so that differences in efficiency could not be attributed to distractor properties. Importantly, observers did not know which target face (i.e., positive or negative) would be presented in a given trial. Differences in search function slopes for positive and negative faces were then examined, revealing that negative faces were searched for more efficiently than positive faces. Eastwood et al. argued that their findings supported the more general conclusion that emotional valence is available preattentively and is able to influence the deployment of attention, as indicated by the differences in efficiency under the experimental conditions employed. It is important to note that such a claim does not exclude the possibility that serial search processes are involved as well, but simply advances the notion that preattentive processes are involved.

Several investigations involving facial stimuli have provided support for the hypothesis that the visual system may be preattentively sensitive to emotional valence and that this may determine subsequent attentional allocation (for a review, see Frischen, Eastwood, \& Smilek, in press). However, these studies have often been criticized on the grounds that alternative interpretations can account for their findings. For example, studies comparing happy and angry face targets using photographic (e.g., Hansen \& Hansen, 1988; Horstmann \& Bauland, 2006) and schematic (e.g., Fox et al., 2000) face images have found more efficient search for angry targets set among happy distrac- tors than vice versa. However, because these studies did not use consistent distractor contexts, it is possible that the results are attributable to distractor properties (e.g., see Hampton, Purcell, Bersine, Hansen, \& Hansen, 1989).

Williams, Moss, Bradshaw, and Mattingley (2005) also cite evidence for different search efficiencies for target faces; these efficiencies depend on emotional expressions. However, although these authors varied set size in their experiment, they based their interpretation on RT differences collapsed across set sizes, rather than on differences in search function slopes, so their data cannot provide evidence for the presence of attentional guidance. It is possible that Williams et al.'s findings reflect differences in RT that result from processes that occur after the target becomes the focus of attention, which would not be expected to vary with set size. Various other investigators have found evidence for facilitated detection of emotional faces in crowds of distractors (e.g., Byrne \& Eysenck, 1995; Fox \& Damjanovic, 2006; Juth, Lundqvist, Karlsson, \& Öhman, 2005; Lundqvist \& Öhman, 2005; Schubö, Gendolla, Meinecke, \& Abele, 2006), but these studies did not involve set size variance, which again makes the findings difficult to interpret.

Several recent studies (Eastwood et al., 2001; Eastwood et al., 2005; Horstmann, Scharlau, \& Ansorge, 2006; Öhman, Lundqvist, \& Esteves, 2001; Suslow et al., 2004; Suslow, Junghanns, \& Arolt, 2001; Suslow, Roestel, Ohrmann, \& Arolt, 2003; White, 1995) have used variable set sizes while holding distractor contexts constant. Many of these studies (Eastwood et al., 2001; Eastwood et al., 2005; Suslow et al., 2004; Suslow et al., 2001; Suslow et al., 2003) have found more efficient search for negatively than for positively valenced schematic face targets set among neutral distractors. Öhman et al. did not find more efficient search for negative faces; however, an examination of their accuracy data shows decreasing accuracy for increasing set sizes with positive distractors, suggesting a speed-accuracy trade-off in the direction predicted by a hypothesis of more efficient search for negative faces (Eastwood \& Smilek, 2005).

It is important, however, that not all studies have shown more efficient search for negative than for positive faces in a neutral distractor context. White (1995) found no differences between search slopes for positive and negative faces in his study, but because the slopes he obtained were flat, he determined that "no firm conclusions can be drawn" (p. 455). More definitively, the study conducted by Horstmann et al. (2006) failed to yield slope differences for positive and negative faces, despite yielding positive search slopes for each target, suggesting that a search advantage for negative faces is not always observed.

The results reviewed so far have typically been interpreted in terms of emotional valence; however, several investigators have questioned the validity of such interpretations (e.g., Horstmann \& Bauland, 2006; Nothdurft, 1993; Purcell \& Stewart, 2006; Purcell, Stewart, \& Skov, 1996). For example, Nothdurft provided evidence that a slope difference obtained in his study between faces expressing various emotions resulted from differences in the shapes of the mouths between two stimuli producing the 
effect, rather than the emotional differences between the targets per se. Furthermore, Purcell, Stewart, and Skov attempted to replicate the finding of Hansen and Hansen (1988) and found that the efficiency differences these authors observed occurred only when study participants were aware of a dark area, in the angry stimulus, which resulted from the transformation of the original grayscale image into black and white, suggesting that this physical characteristic acted as a confound. These authors failed to replicate the effect using nonconfounded grayscale images as stimuli.

Such findings raise the question of whether the effects of facial emotion on attentional allocation are indeed the result of emotional information that guides attention, or whether there are simply nonemotional perceptual attributes contained in targets that yield the observed slope differences. Some physical characteristics that are available preattentively may be consistently present in faces that display a particular emotion, and may also aid in the allocation of attention to targets containing them. White (1995) suggested that such searches may not be referred to as being based on emotion, because one cannot be certain that targets are being attended due to their representation as emotionally meaningful entities, rather than as purely perceptual entities.

The question of which of the observed effects, if any, are attributable to emotional rather than physical attributes has been addressed in different ways. For example, some authors have adopted measures known to adversely affect holistic face perception in an effort to show that such perception is a necessary precondition for the effects they are examining. Vertical inversion of faces, for example, is known to adversely affect facial emotion identification (e.g., Fallshore \& Bartholow, 2003; McKelvie, 1995). Some investigators (Eastwood et al., 2001; Fox et al., 2000) inverted the faces in their visual search studies and found that slope differences, which they had attributed to differences in target emotional valence, disappeared. The importance of the stimuli being upright in order to observe these effects suggests that some holistic quality of the faces contributed to the differences in search efficiency. It is more difficult to interpret these findings in terms of the disruption of the perception of physical properties that are largely preserved in the inverted stimuli than in terms of emotional perception of the targets, which is clearly disrupted by inversion.

It is important, however, that other researchers have obtained contradictory findings. For example, Horstmann and Bauland (2006) used upright or inverted Thatcherized faces, in which individual facial features are inverted but their spatial relations within the face remain unchanged (Thompson, 1980), and observed more efficient search for angry than for happy faces, regardless of their orientation. This result is consistent with a physical properties interpretation of their observed slope differences. White (1995) also inverted the positive and negative schematic faces in his study, but he found the same equivocal results he had obtained with upright faces.

Another control strategy involves assessing the effects of individual facial components on attention allocation.
Some investigators (Fox et al., 2000; Horstmann \& Bauland, 2006) performed control tasks in which only mouth images (which were critical in conveying the emotional meaning of faces in their original task) were presented in order to rule out physical property explanations that relied on the shapes of the mouths themselves. In Fox et al., the valence-contingent RT differences disappeared, supporting emotional-meaning-based interpretations by demonstrating that these components themselves, when removed from a meaning-producing face context, are not responsible for differences in attentional allocation. In Horstmann and Bauland, however, photographs of happy and angry mouths themselves were shown to yield more efficient search for angry targets, much like whole-face photographs. Finally, a study by Purcell and Stewart (2006) showed a search advantage for stimuli that were not angry, but that contained similar physical properties to angry schematic faces.

Taken as a whole, the existing findings are mixed. Employing strategies for eliminating physical-attribute-based explanations of search efficiency has sometimes (but, notably, not always) supported meaning-based interpretations. However, even such support, when it is found, is necessarily equivocal. For example, inversion strategies have demonstrated that some configural quality of these faces that is affected by inversion contributes to the search results. However, emotional valence is only one such quality. Judgments of basic physical qualities of faces, such as spatial relations between elements, are also affected by inversion (e.g., Yasuda, 2005). Inverting face stimuli as a method of control, then, yields results that may still be interpreted in terms of differences between targets that are unrelated to emotional expression. Furthermore, the "feature-only" control strategy may rule out only physical property confounds that are related to facial features themselves, rather than confounds that are related to their presence in some configuration of items.

Given the issues raised here, it is evident that physical confound explanations may be mounted for attentional effects of any stimuli that physically differ in some manner, making the task of uncovering unequivocally valencedriven effects very difficult. Controls that preserve all of the physical characteristics - including the configural properties - of target stimuli may be necessary in order to demonstrate the hypothesized inadequacy of interpretations that are based on the physical properties of the stimuli. This appears to be an impasse, because faces that differ in their emotional expression must differ in their physical properties.

However, another possibility for controlling potential confounds due to the physical characteristics of emotional stimuli involves the assignment of different meanings to otherwise neutral, physically identical stimuli among different observers. This way, physical qualities of stimuli may be held constant while meaning is varied. The potential utility of applying this method to visual search may be seen in other attentional tasks. Several investigators have used stimuli with conditioned threat values in cuing tasks (e.g., Beaver, Mogg, \& Bradley, 2005; Koster, Crombez, Van Damme, Verschuere, \& de Houwer, 
2005; Sonuga-Barke, de Houwer, de Ruiter, Ajzenstzen, \& Holland, 2004; Stormark, Hugdahl, \& Posner, 1999; Van Damme, Crombez, Hermans, Koster, \& Eccleston, 2006), testing Öhman's (1993) hypothesis that humans may be able to use threat-related information to determine how attention should be allocated. For example, Koster et al. (2005) conditioned an aversive noise blast to otherwise neutral stimuli (colored rectangles), and a second set of stimuli were not conditioned. The stimuli were then presented as cues in a task in which the cue either validly or invalidly predicted the side of a fixation cross on which a subsequent probe stimulus was presented. Attentional capture was operationally defined as faster responding to the probe in validly cued trials than in invalidly cued trials. The attention-capturing effect of the aversion-conditioned cues was greater than that for the unconditioned cues, indicating that the acquired meaning of these stimuli had an effect on attentional processes. In summary, a growing body of research has now demonstrated the general principle that when cues have a conditioned threat-signaling value, their attention-capturing qualities change relative to the case in which these stimuli are unconditioned or are paired with benign stimuli.

Two groups have attempted to extend the conditioning methodology to tasks involving targets and distractors. Roskos-Ewoldsen and Fazio (1992) manipulated a variable known as attitude accessibility (Fazio, 1989) by having observers generate evaluative (like-dislike) judgments to one half of a set of 108 schematic stimuli that depicted familiar objects while making living-nonliving judgments for the other half. The objects that had, by random assignment, received evaluative judgments produced greater interference when presented peripherally and irrelevantly in an ongoing, attention-demanding task. These stimuli were also more likely to be detected in a task in which they were presented among a fixed number of distractors.

Batty, Cave, and Pauli (2005) applied an aversionconditioning procedure to complex geometric stimuli, which then served as targets in a visual search task. These authors found no effect of the aversion training on search efficiency. Although this finding might imply that visual search processes are not susceptible to the effects of aversion conditioning, it is possible that these authors simply found a case in which emotional-meaning-based search does not operate. Some qualities of the stimuli these investigators used may have prevented generalizability of their findings; for example, this task required participants to engage in very difficult searches for geometric shapes defined by the configuration of elements. Perhaps there are boundaries for the kinds of physical information that may be used to preattentively signal the visual system to the location of a threat. Unfamiliar, complex shapes may not be processed as efficiently outside of the focus of attention as may be necessary for revealing the potentially subtle effects of emotional valence. It is also possible that complex shapes do not acquire conditioned threat values with the degree of potency required for revealing such effects. The present study, then, is an attempt to expand on Batty et al.'s pioneering work.

\section{THE PRESENT EXPERIMENTS}

In the present investigation, we aim to determine whether preattentive visual processes involved in search are sensitive to emotional valence, so that it may affect the allocation of attention. We also seek to determine the extent to which emotional valence determines attention allocation. The inconsistency in previous findings may simply reflect a small effect of emotional valence that is easily lost amidst the noise of other determinants, such as physical properties.

It is possible that the use of faces in a visual search task will yield different results from those obtained in the experiments of Batty et al. (2005), because faces are more familiar than the geometric shapes used in that investigation, and faces might be processed more efficiently outside the focus of attention. In our experiments, identical sets of face stimuli were used to represent threatening and peaceful targets. Different emotional meanings were conditioned to these target faces among different groups of observers. This way, the physical properties of the target faces remained constant. Therefore, any differences in search efficiency between targets with different conditioned emotional valences suggests that valence is available preattentively and is able to inform attentional allocation.

Several additional methods were included in the present experiments in an attempt to create the conditions under which attentional deployment that is based on emotional valence may be observed. First, a passive search strategy was induced in participants through an instruction set designed by Smilek, Enns, Eastwood, and Merikle (2006). This strategy involves releasing effortful cognitive control over the search process and passively allowing targets to become evident. Smilek, Dixon, and Merikle (2006) found effects of trained categorical meaning on visual search only when they induced a passive search strategy in their observers. Second, the present data are analyzed by experimental block to examine the possibility that time-sensitive effects exist. This is also based on the findings of Smilek, Dixon, and Merikle, who observed that categorical meaning effects in their search tasks were only evident in later blocks of trials; it was proposed that the necessary passive search strategy was achieved to a greater extent in the second half of the experiment. Third, the effects of extinction of target-meaning associations were mitigated in the present study by interleaving search trials with conditioning blocks that were designed to strengthen associations.

Furthermore, our investigation offers a methodological improvement by pooling the results from a series of similar experiments in a meta-analysis. In an effort to achieve a more valid estimate of the potentially small effect size of emotional valence on search, effect size estimates were generated from three experiments. Meta-analysis then offered the twin benefits of producing a more reliable population-level effect size estimate and ensuring the replicability of the findings. Such benefits are especially favorable in the present research context, because previous findings have inconsistently favored either valence-based or physical-property-based interpretations, opening the 
possibility that emotional valence may have a small effect on search that is easily overshadowed by the contributions of physical properties. Performing a single experiment in this case would merely continue the trend of yielding possibly spurious results and would not provide the stable estimate of effect size that is necessary for addressing the issue of inconsistent findings in the literature.

\section{EXPERIMENT 1}

Experiment 1 was designed to demonstrate that efficiency in a visual search task may be affected by the conditioned emotional valence of target face stimuli. Differences in efficiency according to target valence would suggest that this information is available to observers preattentively, that it is able to determine subsequent attention allocation, and that its availability is not solely contingent on physical characteristics.

\section{Method}

Participants. Forty-four York University undergraduate psychology students participated in this experiment in exchange for course credit. Four participants were excluded due to their inability to finish the training procedure in less than $1 \mathrm{~h}$. Of the 40 participants whose data were included in the analyses, 20 were female and 20 were male. All participants reported either normal or corrected-tonormal vision.

Design. The independent variables in this study were target valence (positive, negative) and set size $(4,7,10,13)$. These variables were manipulated within-participant. Between participants, the assignment of valence to target stimuli (henceforth, the trainedvalence variable) was counterbalanced and then collapsed during data analysis. The crucial dependent variable was RT, defined as the time from presentation of the stimulus array to detection of the target, as indicated by a keypress. The other dependent variable measured was response accuracy.

Stimuli. The stimuli used in this study were seven grayscale images of male faces. The faces were chosen from the AR Face Database (Martinez \& Benavente, 1998) in a procedure designed for selecting target and distractor faces that were as emotionally neutral as possible, and for selecting targets that looked physically similar enough to each other to minimize any variability in search slopes that was based on physical properties alone. Twenty-three faces were initially selected by eliminating from the full set the faces that were clearly not neutral. These were then edited in order to eliminate physical properties that might produce pop-out; for example, they were all given identical hairstyles. Two of the faces were then chosen arbitrarily as distractors, and the remaining 21 were designated as targets. In order to select the one distractor and 6 targets to be used in the experiments, a pilot visual search study was conducted. A group of 24 participants performed a visual search task in which they were asked simply to search for the singleton face in each display. Search slopes were then calculated for each target-distractor pair. Participants were also asked to indicate whether they felt each target and distractor looked peaceful or hostile. The final 6 targets and one distractor (see Figure 1) were chosen because of their emotional neutrality and the similarity of their search slopes.

Procedure. Two basic procedures were combined in this experiment. The training procedure involved training the associative emotional labels to the target face stimuli. This procedure comprised a conditioning phase and a test phase.

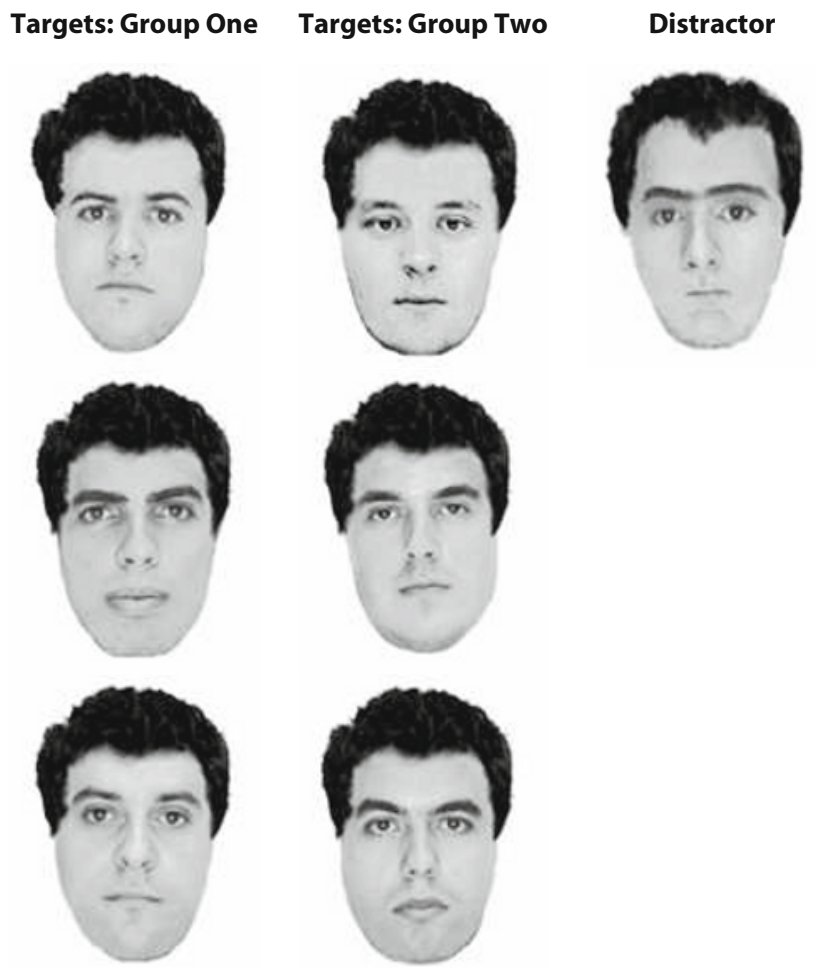

Figure 1. Target and distractor faces used in the three experiments. Targets are shown in the grouping system used to assign one of two emotional labels to each face. 
In the conditioning phase, each target face was presented with its respective emotional label for $1,500 \mathrm{msec}$, with an 800 -msec interval between presentations. Within each group of participants, three faces were paired with the label "peaceful" (positive valence) and three with the label "hostile" (negative valence). Each face-label pair was presented in random order twice per conditioning phase, for a total of 12 presentations per phase.

The test phase was designed for testing the associations established in the conditioning phase and reinforcing the emotional attributions that resulted. In this phase, faces were presented alone, and participants were asked to make a keypress corresponding to the label that had been paired with that face. Face images were displayed until the participant made a response. The keypress was accompanied by a vocal response, a means of ensuring that the target stimulus was conditioned to the label itself, as opposed to the keypress alone. Participants were then provided with accuracy feedback, which remained visible for $1,500 \mathrm{msec}$. Each face was presented twice per test phase, in random order, regardless of participant response accuracy, for a total of 12 presentations per phase.

The second basic procedure was the visual search task itself. In this task, participants were shown a central fixation cross for $500 \mathrm{msec}$. On its offset, the stimulus array appeared (see Figure 2 for an example of such an array). This array consisted of one of the target faces and several distractor faces. Distractor items were homogeneous. Face images in the visual search task subtended approximately $1.2^{\circ} \times 1.7^{\circ}$ of visual angle. The entire array of search items subtended approximately $12.6^{\circ} \times 18.4^{\circ}$ of visual angle. Sets included $4,7,10$, or 13 items, including the target and distractors. The positions of these items were selected randomly from 48 possible positions arranged in a matrix of six rows and eight columns. Spaces not occupied by a stimulus in a given trial were left blank.

Participants were instructed to press the space bar once they identified the unique image on a given trial. After this, participants pressed a key corresponding to the emotion associated with the target presented in that trial: "P" for peaceful targets and " $\mathrm{H}$ " for hostile targets. Emotion identification was, again, accompanied by a vocal statement of the label paired with the target that was presented on that trial. This vocal response served to mitigate extinction of the emotional association, which could occur during the search task. Accuracy feedback was then provided and remained visible for $600 \mathrm{msec}$. The next trial then began automatically after a delay of $300 \mathrm{msec}$. See Figure 3 for a depiction of the time course of the search task.

Course of the experiment. The experiment began with a brief orientation about the general course of the experiment, followed by obtaining written informed consent. Participants then performed iterations of the training procedure until they achieved $92 \%$ accuracy (11 accurate identifications out of 12 test trials) five times. This was done to ensure that participants could accurately identify faces before beginning the search trials and to reinforce the associations formed for face-label pairs.

Participants then advanced to the search trials, performing one block of 36 such trials as practice. Following the practice trials, three blocks of experimental search tasks were completed. Each block contained 48 trials: 6 trials for each set of variables resulting from a full crossing of target emotion (two levels) and set size (four levels). Between experimental blocks, participants completed one additional training procedure in order to mitigate extinction of the associative pairings.

Prior to the search trials, participants were also given instructions designed to induce a passive search strategy, in order to relax the cognitive control over eye movements during search (Smilek, Dixon, \& Merikle, 2006; Smilek, Enns, et al., 2006). The instructions were as follows:

Advice for completing the search task: The best way to search for the unique face is to sit back and let your eyes find it for you, rather than actively directing your gaze at each face, one after another. You'll find at some point it will pop out without much conscious effort.

This procedure was selected because the passive strategy is hypothesized to allow participants to conduct search based on conditioned emotional valence, avoiding the use of a search strategy that has, in a prior study, obscured the effects of stimulus meaning (Smilek, Dixon, \& Merikle, 2006; for further discussion of the cognitive effects of passive viewing strategies, see Lleras \& Von Mühlenen,

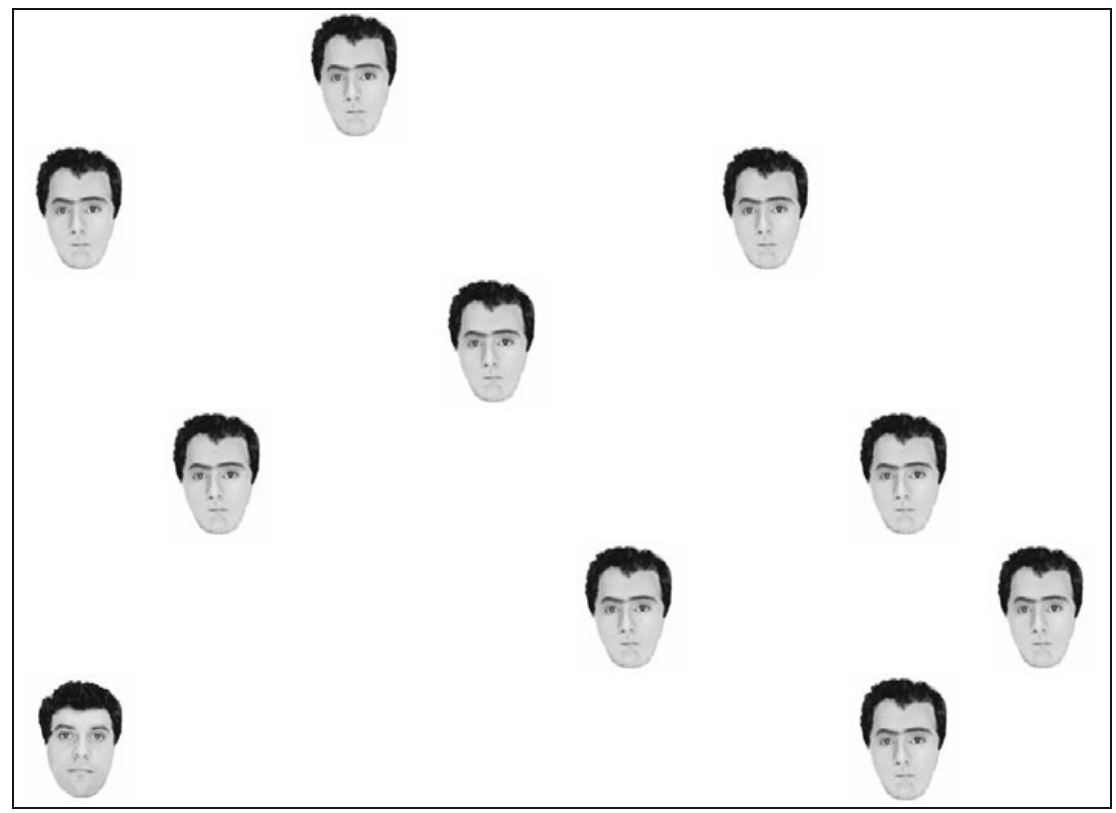

Figure 2. Example of a 10-item stimulus array in Experiment 1. The target is in the lower left-hand position. 


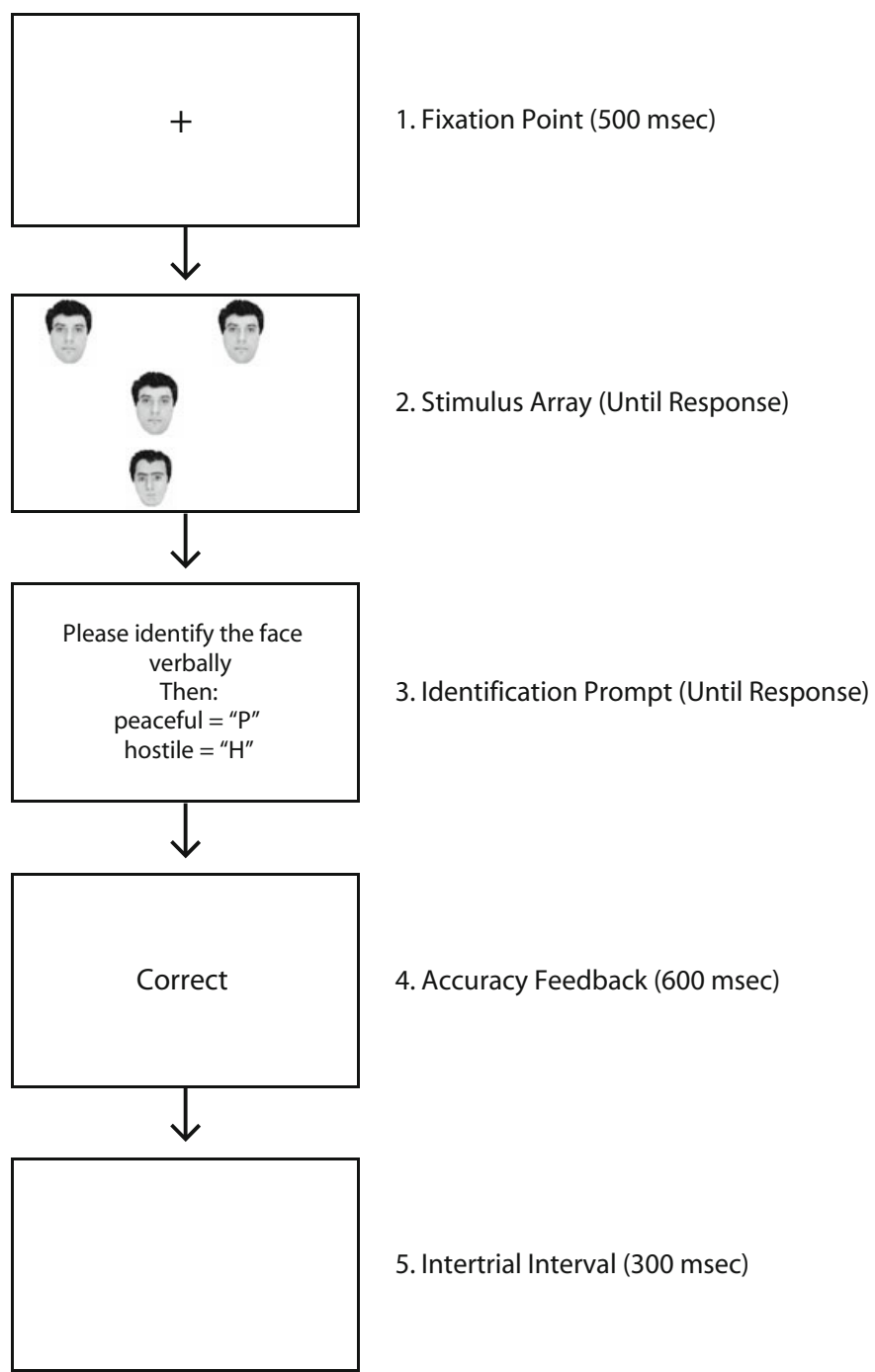

Figure 3. Sequence of events in each trial in the search task.

2004; Marcel, 1983; Snodgrass, Shevrin, \& Kopka, 1993; Van Selst \& Merikle, 1993).

The experiment took between 30 and $60 \mathrm{~min}$ for participants to complete and was followed by a debriefing, in which we explained the purpose of the study.

\section{Results}

Participants completed an average of 11 iterations of the training procedure before beginning the search procedure. Data from across all three blocks of search trials were analyzed first. A second set of analyses was then performed to determine whether a different pattern of search performance could be observed in the final two blocks of trials. The data for the RT analyses consisted of search slopes, in milliseconds per display item, with the RTs from error trials removed. In all, $3.9 \%$ of the trials were excluded from analysis due to inaccurate responding. Error rate data were also transformed into slopes and analyzed in order to examine the possibility that linear changes in RTs across different set sizes may coincide with linear changes in accuracy, suggesting that RT changes may be accounted for by a speed-accuracy trade-off.

Performance across all experimental blocks. The difference in RT slopes for peaceful and hostile targets was analyzed using a two-tailed, repeated measures $t$ test. A nonsignificant trend toward greater average search slopes for peaceful (105 msec/item) than for hostile (92 msec/item) targets was observed $[t(39)=1.78, p=$ $.08]$. Accuracy data were also analyzed using a two-tailed, repeated measures $t$ test. No significant difference was found between accuracy slopes for peaceful and hostile targets $[t(39)=-0.29, p=.77]$.

Performance in Blocks 2 and 3 examined alone. In order to examine effects that may only be evident in later experimental trials (as in Smilek, Dixon, \& Merikle, 
2006), RTs and accuracy in Blocks 2 and 3 were analyzed using two-tailed, repeated measures $t$ tests and are displayed as a function of set size and target emotion in Figure 4 . The average search slope for hostile faces $(84 \mathrm{msec} /$ item) was shallower than that for peaceful faces $(104 \mathrm{msec} /$ item) $\left[t(39)=2.16, p=.04, r^{2}=.11\right]$. This result suggests different search efficiencies for targets depending on their emotional valence. Accuracy data corresponding to these RTs are presented in the bar graph component of Figure 4. No significant difference was found between accuracy slopes for peaceful and hostile targets, two-tailed $[t(39)=-0.29, p=.78]$.

\section{Discussion}

The results from this experiment and, in particular, those from the final two blocks of trials, are consistent with the hypothesis that emotional meaning, independent of physical stimulus properties, is preattentively available and may affect the allocation of attention. More efficient search for threatening targets was shown in these blocks by the shallower search slope for targets that were associated with a threatening label.

This emotion-contingent difference in search efficiency cannot be attributed to physical differences among stimulus sets because such differences were counterbalanced during data aggregation. Consistent physical differences among stimuli exemplifying various emotional meanings were eliminated in this way, because data for each different target emotion were collected with equal numbers of the same face images.

The difference in search slopes for the two target emotions has a small estimated effect size $\left(r^{2}=.11\right)$. The limited variance accounted for by target emotion is consistent

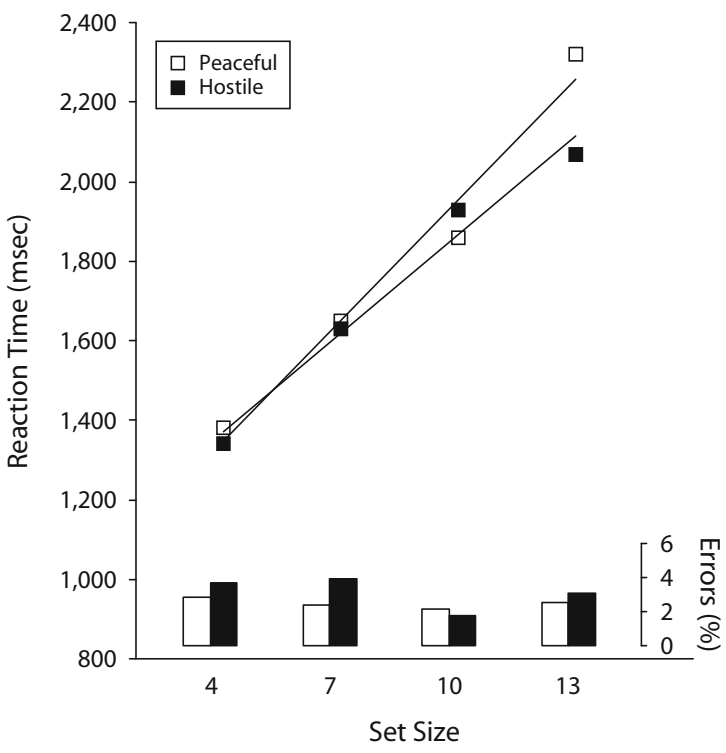

Figure 4. Mean reaction times and percentages of error for identifying the peaceful and hostile faces in Experiment 1, Blocks 2 and 3 only. with the previous inconsistency of findings in the prior literature, and suggests that the present findings require replication in order to be compelling.

\section{EXPERIMENT 2}

The primary purpose of Experiment 2 was to replicate the basic finding of Experiment 1, in which different search efficiencies were observed for the two target emotional meanings. In an attempt to increase the effect of emotional valence, the visual angle subtended by stimuli was increased in Experiment 2. This manipulation was intended to make the search task easier for participants. The steep search function slopes yielded in Experiment 1 suggested a difficult search task that may not have been sufficiently sensitive to effects of target emotion.

\section{Method}

Participants. Fifty-one York University undergraduate psychology students volunteered to participate in this experiment in exchange for course credit. Five participants were excluded due to their inability to finish the training procedure in less than $1 \mathrm{~h}$. Of the 46 participants whose data were included in analyses, 32 were female and 14 were male. All participants reported either normal or corrected-to-normal vision.

Stimuli and Procedure. The design and stimuli used in this experiment were identical to those used in Experiment 1, except for two changes. In this experiment, we presented sets of 4,8 , or 12 stimuli positioned in a matrix of four columns and three rows. The stimuli presented here were larger than those in Experiment 1, subtending approximately $4.0^{\circ} \times 2.9^{\circ}$ of visual angle. The entire array of search items subtended $13.6^{\circ} \times 17.6^{\circ}$ of visual angle.

The procedures followed in this experiment were identical to those followed in Experiment 1, except that after the practice trials, four blocks of experimental search tasks were completed, rather than three. Each block contained 36 trials: 6 for each set of variables resulting from a full crossing of target emotion (two levels) and set size (three levels). As in Experiment 1, participants completed one additional training phase and one additional test phase between blocks of visual search trials.

\section{Results}

The same data analysis procedures followed in Experiment 1 were followed here. In all, $3.1 \%$ of the trials were excluded from analysis due to inaccurate responding. Participants completed an average of 10.1 iterations of the training procedure before beginning the search procedure.

Performance across all experimental blocks. No significant difference was found between average search slopes for hostile (66 msec/item) and peaceful $(73 \mathrm{msec} /$ item) targets [two-tailed $t(45)=0.92, p=.36$ ]. Likewise, no difference was evident in accuracy slopes between emotion and set size [two-tailed $t(45)=0.32, p=.75$ ]

Performance in Blocks 3 and 4 examined alone. RT and accuracy performance for Blocks 3 and 4 are displayed in Figure 5. The average search slope for hostile faces ( $64 \mathrm{msec} /$ item) was shallower than that for peaceful faces $(82 \mathrm{msec} /$ item), [two-tailed $t(45)=2.50, p=.02$, $\left.r^{2}=.12\right]$. Accuracy data corresponding to these RTs are presented in the bar graph component of Figure 5. No difference was evident in accuracy slopes for peaceful and hostile targets [two-tailed $t(45)=0.67, p=.51$ ]. 


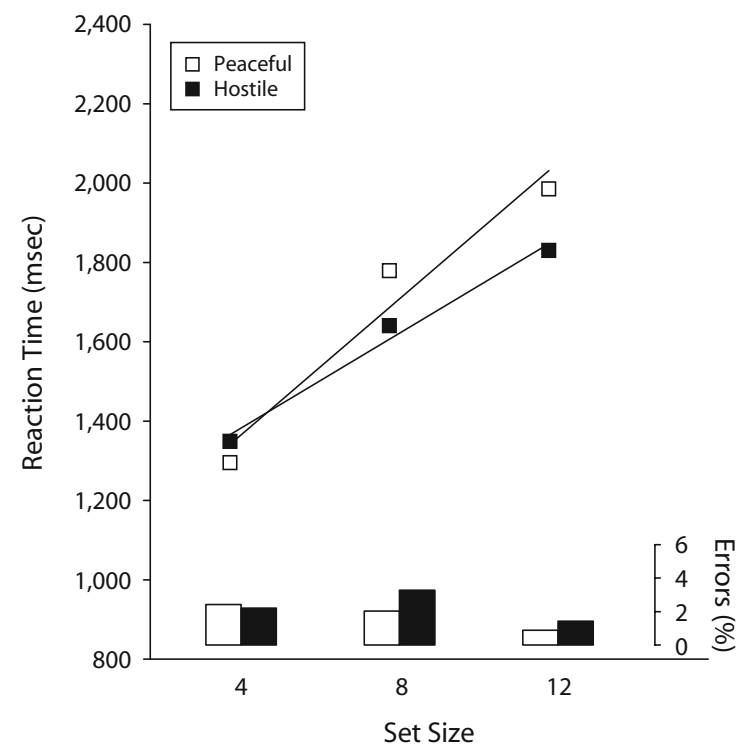

Figure 5. Mean reaction times and percentages of error for identifying the peaceful and hostile faces in Experiment 2 , Blocks 3 and 4 only.

\section{Discussion}

The results of this experiment replicated those of Experiment 1 , yielding a significant difference between search efficiencies for peaceful and hostile targets in the second half of the experiment. As in Experiment 1, these results may not be attributed to physical differences between faces conveying various emotions, differing distractor contexts, or trade-offs between speed and accuracy.

\section{EXPERIMENT 3}

Experiment 3 was a replication of Experiment 2 and followed an identical procedure. Further replication was deemed necessary, due to the small effects observed in Experiments 1 and 2. Given the inherent difficulty in observing small effects, this replication offered an opportunity to estimate more accurately the size of the effect and to examine its robustness. Specifically, adding a third experiment allowed for more effect size estimates and $p$ statistics to be entered into a meta-analysis, conducted subsequently, aimed at obtaining a more stable overall effect size estimate.

\section{Method}

Thirty-seven adults were recruited to participate in this study via posters that were displayed at York University. These participants were given $\mathrm{C} \$ 8.00$ for a maximum of $1 \mathrm{~h}$ of participation. Three participants were excluded due to their inability to finish the training procedure in less than $1 \mathrm{~h}$. Of the 34 participants whose data were included in the analyses, 20 were female and 14 were male. All participants reported either normal or corrected-to-normal vision.

All procedures followed in this experiment were identical to those followed in Experiment 2.

\section{Results}

RT and accuracy data were analyzed following procedures identical to those followed in Experiments 1 and 2 . In all, $2.4 \%$ of the trials were excluded from analysis due to inaccurate responding. Participants completed an average of 9.5 iterations of the training procedure before beginning the search procedure.

Performance across all experimental blocks. No significant difference was found between average search slopes for peaceful $(73 \mathrm{msec} / \mathrm{item})$ and hostile $(77 \mathrm{msec} /$ item) targets [two-tailed $t(33)=-0.64, p=.53$ ], and no difference was evident between accuracy slopes [twotailed $t(33)=-0.13, p=.90]$.

Performance in Blocks 3 and 4 examined alone. RT and accuracy performance for Blocks 3 and 4 are displayed in Figure 6. No significant difference was found between average search slopes for peaceful $(61 \mathrm{msec} /$ item) and hostile (70 msec/item) targets in Blocks 3 and 4 [two-tailed $t(33)=-0.82, p=.42$ ] and no difference was evident in accuracy slopes [two-tailed $t(33)=0.92$, $p=.37]$.

\section{Discussion}

The results of Experiment 3 failed to replicate the findings of Experiments 1 and 2. No differences in search efficiency were observed in the present experiment, despite the fact that the procedure followed was identical to that of Experiment 2. This replication failure is consistent with the mixed results of previous studies that attempted to provide support for interpretations based on physical attri-

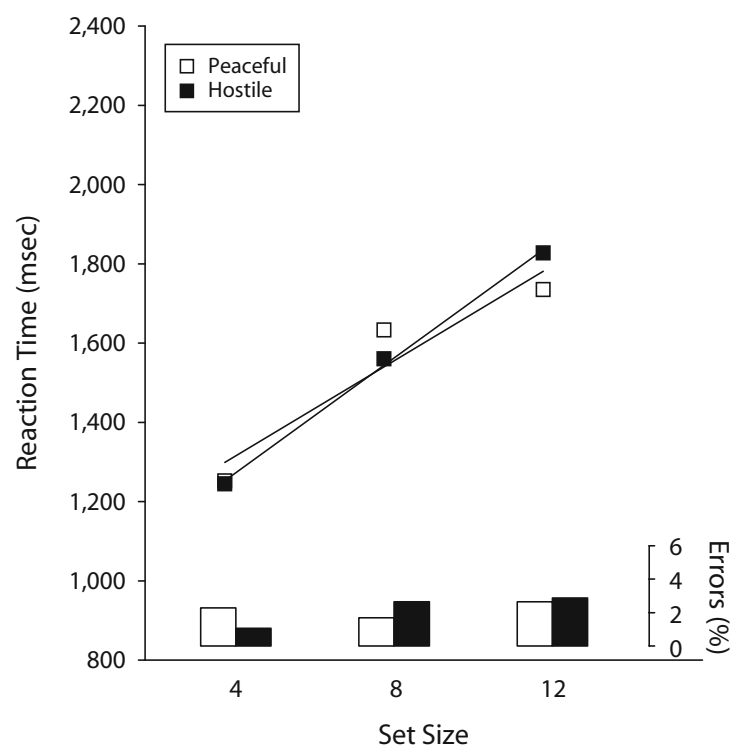

Figure 6. Mean reaction times and percentages of error for identifying the peaceful and hostile faces in Experiment 3, Blocks 3 and 4 only. 
butes or emotional meaning. Three possible explanations exist for the current failure to replicate: (1) It could be due to differences between the populations under study (e.g., paid vs. unpaid); (2) there could be no real populationlevel effect, and the results of Experiments 1 and 2 could be spurious; or (3) there could be a real population-level effect that is not always detected, due to chance sampling variation. It is hypothesized that our mixed results represent a real effect of emotional meaning that is inconsistently detected, due to its relatively small size. The most suitable method for resolving this ambiguity and assessing the suggested hypothesis is meta-analysis.

\section{META-ANALYSIS OF EXPERIMENTS 1, 2, AND 3}

In order to calculate an estimate of the population-level effect size for emotional valence on attentional orienting, and to ensure that the effect is significant at the population level, a meta-analysis was performed. Given the small effects observed in Experiments 1-3 and the inconsistency observed in findings across these and other experiments assessing meaning-based visual search, it may be hypothesized that there exists a small but significant effect of target emotional valence on visual search. The present meta-analysis allows us to test that hypothesis. In keeping with the observation that valence-based effects emerged in the later blocks of each experiment, only data from these blocks were entered into the meta-analysis.

\section{Method}

A pooling of effect size and $p$-level values was performed as outlined by Rosenthal (1991), whose methods were followed throughout the meta-analysis.

Pooled significance. In order to determine whether the overall effect observed across the later experimental blocks of Experiments 1-3 was statistically significant, or whether the findings of the first two experiments were most likely spurious, a significance test was performed. First, the $p$ value from each experiment was transformed into a corresponding $z$ score. A weighted mean (according to the sample size of the respective experiments) of these $z$ scores was then calculated. The weighting scheme was intended to give precedence to larger studies, which provide better estimates of population-level effects. The $z$ score for Experiment 3, which yielded effects in the opposite direction to Experiments 1 and 2, was assigned a negative value. Once the weighted mean of the $z$ scores was calculated, it was transformed into a $p$ value that provided an estimate of the likelihood that the pattern of results obtained across the three experiments came about by chance (and did not reflect a population-level difference in search efficiency for hostile and peaceful targets). The hypothesis that target emotion is available preattentively and may determine subsequent deployment of attention would be considered supported if there was a significant overall difference in search slopes for peaceful and hostile targets across the three experiments.

Pooled effect size estimate. The calculation of the pooled estimate of effect size involved several steps intended to yield an unbiased and appropriately weighted mean value. An estimate of the variance in search slopes accounted for by target emotion $(r)$ was first calculated for each experiment. The $r$ values were then transformed, as suggested by Fisher (1928), in order to correct for bias, producing $z_{r}$ scores. The $z_{r}$ scores were then weighted according to the sample size of each experiment, again giving precedence to larger studies, which provide better estimates of population-level effects. The $z_{r}$ score for Experiment 3, with its reverse effect, was again assigned a negative value. Weighted scores were then averaged, and the weighted mean of these scores was transformed into a pooled estimate of $r^{2}$, the percentage of the variance in search slopes accounted for by target emotion across the three experiments. This $r^{2}$ value provides a measure of the effect of target emotion on search efficiency.

\section{Results and Discussion}

The weighted mean of $z$ scores in the pooled significance test was significant $(z=2.36, p=.018)$. This $p$ value describes the likelihood that the overall difference in search slopes for peaceful and hostile targets observed across the present experiments was obtained by chance.

The weighted mean of the corrected effect size estimates $\left(z_{r}\right.$ scores $)$ for the three experiments was .21, corresponding to an estimated $r$ value of .21 and an $r^{2}$ value of .04. This indicates that $4 \%$ of the variance in search efficiency in these experiments was accounted for by target emotion.

These results demonstrate more efficient search for hostile targets than for peaceful targets in the later blocks of trials of each experiment. The results support the hypothesis of a small but significant effect of target emotional valence, which evidently accounts for only a small portion of the variability in search efficiency across the present experiments. A small effect such as this may be expected to yield occasional null results. It also indicates the relative importance of factors other than emotional valence, such as physical stimulus properties, in determining attentional allocation in visual search.

\section{GENERAL DISCUSSION}

Taken together, the results of the present experiments and meta-analysis go a long way toward settling a growing debate regarding the relationship between emotion and attention. Specifically, there has been considerable debate concerning whether emotional meaning in faces can guide attention. Some authors (e.g., Horstmann \& Bauland, 2006; Horstmann et al., 2006; Nothdurft, 1993; Purcell \& Stewart, 2006; Purcell et al., 1996) have presented findings in favor of physical stimulus properties as the sole determinants of attention deployment, whereas others (e.g., Eastwood et al., 2001; Eastwood et al., 2005; Fox et al., 2000; Suslow et al., 2004; Suslow et al., 2001; Suslow et al., 2003) have presented findings that suggest a role for emotional valence per se. The present study resolves this debate by demonstrating (1) more efficient search for threatening than for peaceful faces when the target faces were embedded in neutral face distractors and when physical stimulus properties were exactly the same across conditions, and (2) that the magnitude of this effect is small, leaving a great deal of the variance in search efficiency unaccounted for by emotional valence. Therefore, the present findings support the hypotheses of several authors (e.g., Eastwood et al., 2001; Hansen \& Hansen, 1988; Öhman, 1993; Williams et al., 2005) who have argued that the visual system is capable of preattentively using information about the emotional valence of a target item before that item becomes the focus of attention. The present finding of a small effect of valence on 
search efficiency is also consistent with those studies that fail to find any robust influences of emotional valence on attention: Although information about the emotional valence of targets is available preattentively, its contribution to search efficiency in many laboratory studies may be overshadowed by the contributions of physical stimulus properties. Physical stimulus properties may even interact with stimulus meaning; some stimuli may contain physical properties that enhance, preempt, or otherwise affect the role of meaning in search, similar to the interaction summarized by Wolfe (2001) between physical stimulus properties and the role of familiarity in search.

The present investigation demonstrates the value of the conditioned meaning paradigm and the potential for extending its use to visual search methodology by demonstrating that conditioned stimulus qualities influence search efficiency. This result serves to extend Smilek, Dixon, and Merikle's (2006) finding that the learned categorical meaning of a stimulus influences search. In that study, categorical labels were trained to various stimuli (i.e., line segments were given names according to their orientation). When those stimuli were used as targets and distractors in a subsequent search task, the similarity of category membership between target and distractor in search displays influenced search efficiency. The present study expands on this finding by demonstrating search efficiency effects using emotional, rather than categorical, meaning. It also contributes by evaluating the effects on attention of a learned meaning with clearer ethological implications (i.e., threat).

Some questions about the present results remain unanswered. For example, although they clearly demonstrate a role for the emotional meaning of faces in determining visual search efficiency, meaning in this context was based on facial identity, to which emotional valence was conditioned, rather than facial expression per se. Since facial expression is a potent signal conveying emotional meaning, it is likely that target faces with emotional expressions will also be searched for on the basis of their meaning. However, the present results only point toward this possibility and do not demonstrate it; it is possible that the present results cannot be extrapolated to any prepotently meaningful stimulus.

The present findings also do not reveal how meaningbased attentional allocation is executed. It is possible that the meaning of a target stimulus itself is processed preattentively; however, it is also possible that meaning is, through learning, connected with particular physical properties that are then searched for preferentially. According to the latter account, the target stimuli are not selected on the basis of emotional valence, as appraised within any given search trial, but according to physical properties that were associated with valence in previous trials. Thus, preattentive identification of valence might not occur, despite targets' being preferentially attended, due to their meaning. ${ }^{1}$

Another limitation of the present experiments is that they did not seek to parse out processes that occur after a target has been fixated. It is possible that the present slope differences (or indeed those obtained in any visual search task) are attributable to a process in which targets are fixated on and rejected erroneously as distractors, re- quiring further search behavior until they are refixated. This postattentive account of differential search slopes for face targets is conceivable; however, it has been explored in a study by Reynolds, Eastwood, Partanen, Frischen, and Smilek (in press; see also Smilek, Frischen, Reynolds, Gerritsen, \& Eastwood, 2007), who failed to find any evidence that such processes occur.

Finally, the reason our investigation and that of Smilek, Dixon, and Merikle (2006) yielded results only in later trials warrants further investigation The consistency of this finding across several experiments suggests that it is not coincidental and implicates some underlying cognitive mechanism. Determining what this mechanism is would be useful for understanding meaning-based search. It is possible that in the present investigation, the increased number of exposures to paired stimuli that accumulated toward the end of the experiment strengthened the association between labels and faces, increasing differences in search efficiency for the differently labeled targets. Another hypothetical account involves search strategy. Observers may gradually learn increased passivity - a more efficient search strategy, allowing them to abandon an active strategy that involves more eye movements and/or cognitive control. Smilek, Dixon, and Merikle (2006) suggested this idea on the basis of subjective reports from their participants, who found themselves using such a strategy during the later part of another search experiment. One possible account for the effects of passive search is that passive search instructions delay the first eye movement made by an observer during a search task. Brown et al. (1997) found that one of the participants in their study, who tended to withhold their first saccade much longer than the others, was able to accurately discriminate and locate face images presented peripherally in a crowd, whereas others lacked this ability. This observation suggests a connection between the latency of the first saccade in a search task and one's ability to discriminate complex stimuli preattentively. Active search may decrease the latency of an initial eye movement, disrupting a process that is crucial for the preattentive identification of facial stimuli.

It is notable that the present findings run contrary to the prevailing view that emotional valence cannot determine attentional deployment. For example, in a recent review, Wolfe and Horowitz (2004) concluded that angry faces probably do not guide the deployment of attention. These authors are not alone in considering the emotional properties of faces and other stimuli to be incapable of determining attentional allocation, citing evidence (e.g., Purcell et al., 1996) favorable to physical characteristic accounts of emotion-based search. Although the techniques employed by investigators have heretofore provided possibly confounded answers regarding the ability of facial emotion to determine attention allocation, the present study demonstrates the effect of emotional valence where physical properties per se could not have determined attention allocation across all observers. The present investigation has therefore avoided a major pitfall and shown more conclusively that emotional valence can determine attention allocation.

The present investigation answers the question of whether the visual system is able to allocate attention 
using processes that are driven by the emotional meaning of stimuli, and whether such allocation is solely based on physical properties. However, the findings also question the meaningfulness of emotional valence in determining search performance; that is, in the present experimental context, emotional valence accounted for a very small proportion of variance in search performance. It is possible that altering variables, such as the potency of the emotional valence of a target, may increase the effect of target valence on search efficiency; however, this remains to be examined. If the size of emotional valence effects is consistently small across different experimental contexts, even with potent stimuli, powerful studies will be necessary in order to reveal and investigate them. It should not be assumed that a strong indication of physical property effects on the efficiency of search for emotional faces means that emotional valence is not available preattentively. Inconsistent findings pertaining to meaning-based search may simply be the result of insufficient experimental power, as well as the overwhelming contributions of other factors, such as physical stimulus properties.

\section{Conclusion}

The present investigation demonstrates that emotional information is available preattentively and is able to determine attentional allocation. However, under the conditions employed in this investigation, the role of emotional valence was small. It is important that the present experimental method provided the opportunity to observe the effects of emotional valence on visual search, while eliminating effects that are unrelated to emotional valence and are based purely on physical stimulus properties. This investigation thus clarifies the functioning of the visual attention system in a context involving meaning, bringing our understanding of attention a small step closer to the world outside the laboratory.

\section{AUTHOR NOTE}

The authors thank Elena Chernenko, Jean-Francois Nankoo, and Matthew Dubins for their assistance in conducting these experiments. We also acknowledge Michael Reynolds, who contributed the computer program on which our programs were based, and our reviewers for their insightful comments. Correspondence concerning this article should be addressed to C. Gerritsen, Department of Psychology, York University, 4700 Keele Street, Toronto, ON, Canada M3J 1P3 (e-mail: cjgerrit@ yorku.ca).

\section{REFERENCES}

Batty, M. J., Cave, K. R., \& Pauli, P. (2005). Abstract stimuli associated with threat through conditioning cannot be detected preattentively. Emotion, 5, 418-430.

Beaver, J. D., MogG, K., \& Bradley, B. P. (2005). Emotional conditioning to masked stimuli and modulation of visuospatial attention. Emotion, 5, 67-79.

Brown, V., Huey, D., \& Findlay, J. M. (1997). Face detection in peripheral vision: Do faces pop out? Perception, 26, 1555-1570.

BYRNE, A., \& EYSENCK, M. W. (1995). Trait anxiety, anxious mood, and threat detection. Cognition \& Emotion, 9, 549-562.

Duncan, J., \& Humphreys, G. W. (1989). Visual search and stimulus similarity. Psychological Review, 96, 433-458.

EAstwood, J. D., \& SMILEK, D. (2005). Functional consequences of perceiving facial expressions of emotion without awareness. Consciousness \& Cognition, 14, 565-584.
Eastwood, J. D., Smilek, D., \& Merikle, P. M. (2001). Differential attentional guidance by unattended faces expressing positive and negative emotion. Perception \& Psychophysics, 63, 1004-1013.

Eastwood, J. D., Smilek, D., OAKman, J. M., Farvolden, P., van Ameringen, M., Mancini, C., \& Merikle, P. M. (2005). Individuals with social phobia are biased to become aware of negative faces. $\mathrm{Vi}$ sual Cognition, 12, 159-179.

FALLSHORE, M., \& BARTHOLOW, J. (2003). Recognition of emotion from inverted schematic drawings of faces. Perceptual \& Motor Skills, 96, 236-244.

FAZIO, R. H. (1989). On the power and functionality of attitudes: The role of attitude accessibility. In A. R. Pratkanis, S. J. Breckler, \& A. G. Greenwald (Eds.), Attitude structure and function (pp. 153-179). Hillsdale, NJ: Erlbaum.

FISHER, R. A. (1928). Statistical methods for research workers (2nd ed.). Edinburgh: Oliver \& Boyd.

Fox, E., \& Damuanovic, L. (2006). The eyes are sufficient to produce a threat superiority effect. Emotion, 6, 534-539.

Fox, E., Lester, V., Russo, R., Bowles, R. J., Pichler, A., \& DutTON, K. (2000). Facial expressions of emotion: Are angry faces detected more efficiently? Cognition \& Emotion, 14, 61-92.

Frischen, A., Eastwood J. D., \& Smilek D. (in press). Visual search for faces with emotional expressions. Psychological Bulletin.

Hampton, C., Purcell, D. G., Bersine, L., Hansen, C. H., \& HanSEN, R. D. (1989). Probing "pop-out": Another look at the face-in-thecrowd effect. Bulletin of the Psychonomic Society, 27, 563-566.

Hansen, C. H., \& Hansen, R. D. (1988). Finding the face in the crowd: An anger superiority effect. Journal of Personality \& Social Psychology, 54, 917-924.

Horstmann, G., \& Bauland, A. (2006). Search asymmetries with real faces: Testing the anger-superiority effect. Emotion, 6, 193-207.

Horstmann, G., Scharlau, I., \& Ansorge, U. (2006). More efficient rejection of happy than of angry face distractors in visual search. Psychonomic Bulletin \& Review, 13, 1067-1073.

Juth, P., LundQvist, D., Karlsson, A., \& Öhman, A. (2005). Looking for foes and friends: Perceptual and emotional factors when finding a face in the crowd. Emotion, 5, 379-395.

Kahneman, D., \& Treisman, A. (1984). Changing views of attention and automaticity. In R. Parasuraman \& D. A. Davis (Eds.), Varieties of attention (pp. 28-61). San Diego: Academic Press.

Koster, E. H. W., Crombez, G., Van Damme, S., Verschuere, B., \& DE Houwer, J. (2005). Signals for threat modulate attentional capture and holding: Fear-conditioning and extinction during the exogenous cueing task. Cognition \& Emotion, 19, 771-780.

Lleras, A., \& Von Mühlenen, A. (2004). Spatial context and top-down strategies in visual search. Spatial Vision, 17, 465-482.

LuNDQVIST, D., \& ÖHMAN, A. (2005). Emotion regulates attention: The relation between facial configurations, facial emotion, and visual attention. Visual Cognition, 12, 51-84.

Marcel, A. J. (1983). Conscious and unconscious perception: Experiments on visual masking and word recognition. Cognitive Psychology, 15, 197-237.

Martinez, A. M., \& Benavente, R. (1998). The AR face database (Tech. Rep. No. 24). Barcelona, Spain: Universitat Autònoma de Barcelona, Computer Vision Center.

McKelvie, S. J. (1995). Emotional expression in upside-down faces: Evidence for configurational and componential processing. British Journal of Social Psychology, 34, 325-334.

Nothdurft, H.-C. (1993). Faces and facial expressions do not pop out. Perception, 22, 1287-1298.

Öhman, A. (1993). Fear and anxiety as emotional phenomena: Clinical phenomenology, evolutionary perspectives, and informationprocessing mechanisms. In M. Lewis \& J. M. Haviland (Eds.), Handbook of emotions (pp. 511-536). New York: Guilford.

Öhman, A., LundQvist, D., \& Esteves, F. (2001). The face in the crowd revisited: A threat advantage with schematic stimuli. Journal of Personality \& Social Psychology, 80, 381-396.

Purcell, D. G., \& Stewart, A. L. (2006, May). The anger superiority effect: Confounds with schematic stimuli. Poster presented at the 18th Annual Meeting of the Association for Psychological Science, New York.

Purcell, D. G., Stewart, A. L., \& Skov, R. B. (1996). It takes a confounded face to pop out of a crowd. Perception, 25, 1091-1108. 
Reynolds, M. G., Eastwood, J. D., Partanen, M., Frischen, A., \& SMILEK, D. (in press). Monitoring eye movements while searching for affective faces. Visual Cognition.

Rosenthal, R. (1991). Meta-analytic procedures for social research (Rev. ed.). Thousand Oaks, CA: Sage.

Roskos-Ewoldsen, D. R., \& Fazio, R. H. (1992). On the orienting value of attitudes: Attitude accessibility as a determinant of an object's attraction of visual attention. Journal of Personality \& Social Psychology, 63, 198-211.

Schubö, A., Gendolla, G. H. E., Meinecke, C., \& Abele, A. E. (2006). Detecting emotional faces and features in a visual search paradigm: Are faces special? Emotion, 6, 246-256.

Smilek, D., Dixon, M. J., \& Merikle, P. M. (2006). Revisiting the category effect: The influence of meaning and search strategy on the efficiency of visual search. Brain Research, 1080, 73-90.

Smilek, D., Enns, J. T., Eastwood, J. D., \& Merikle, P. M. (2006). Relax! Cognitive strategy influences visual search. Visual Cognition, 14, 543-564.

SmileK, D., Frischen, A., Reynolds, M. G., Gerritsen, C., \& Eastwood, J. D. (2007). What influences visual search efficiency? Disentangling contributions of preattentive and postattentive processes. Perception \& Psychophysics, 69, 1105-1116.

Snodgrass, M., Shevrin, H., \& KopKa, M. (1993). The mediation of intentional judgments by unconscious perceptions: The influence of task strategy, task preference, word meaning, and motivation. Consciousness \& Cognition, 2, 169-193.

Sonuga-Barke, E. J. S., DE Houwer, J., DE Ruiter, K., Ajzenstzen, M., \& Holland, S. (2004). AD/HD and the capture of attention by briefly exposed delay-related cues: Evidence from a conditioning paradigm. Journal of Child Psychology \& Psychiatry, 45, 274-283.

Stormark, K. M., Hugdahl, K., \& Posner, M. I. (1999). Emotional modulation of attention orienting: A classical conditioning study. Scandinavian Journal of Psychology, 40, 91-99.

Suslow, T., Dannlowski, U., Lalee-Mentzel, J., Donges, U.-S., Arolt, V., \& Kersting, A. (2004). Spatial processing of facial emotion in patients with unipolar depression: A longitudinal study. Journal of Affective Disorders, 83, 59-63.

Suslow, T., Junghanns, K., \& Arolt, V. (2001). Detection of facial expressions of emotions in depression. Perceptual \& Motor Skills, 92, 857-868.

Suslow, T., Roestel, C., Ohrmann, P., \& Arolt, V. (2003). Detection of facial expressions of emotions in schizophrenia. Schizophrenia Research, 64, 137-145.
Thompson, P. (1980). Margaret Thatcher: A new illusion. Perception, 9, 483-484.

Treisman, A. M., \& Gelade, G. (1980). A feature-integration theory of attention. Cognitive Psychology, 12, 97-136.

Tsotsos, J. K. (1997). Limited capacity of any realizable perceptual system is a sufficient reason for attentive behavior. Consciousness \& Cognition, 6, 429-436.

Van Damme, S., Crombez, G., Hermans, D., Koster, E. H. W., \& EcCleston, C. (2006). The role of extinction and reinstatement in attentional bias to threat: A conditioning approach. Behaviour Research \& Therapy, 44, 1555-1563.

Van Selst, M., \& Merikle, P. M. (1993). Perception below the objective threshold? Consciousness \& Cognition, 2, 194-203.

White, M. (1995). Preattentive analysis of facial expressions of emotion. Cognition \& Emotion, 9, 439-460.

Williams, M. A., Moss, S. A., Bradshaw, J. L., \& Mattingley, J. B. (2005). Look at me, I'm smiling: Visual search for threatening and nonthreatening facial expressions. Visual Cognition, 12, 29-50.

Wolfe, J. M. (1989). Visual search. In H. Pashler. (Ed.), Attention (pp. 13-73). Hove, U.K.: Psychology Press.

Wolfe, J. M. (1998). What can 1 million trials tell us about visual search? Psychological Science, 9, 33-39.

Wolfe, J. M. (2001). Asymmetries in visual search: An introduction. Perception \& Psychophysics, 63, 381-389.

Wolfe, J. M. (2003). Moving towards solutions to some enduring controversies in visual search. Trends in Cognitive Sciences, 7, 70-76.

Wolfe, J. M., Cave, K. R., \& Franzel, S. L. (1989). Guided search: An alternative to the feature integration model for visual search. Journal of Experimental Psychology: Human Perception \& Performance, 15, 419-433.

Wolfe, J. M., \& Horowitz, T. S. (2004). What attributes guide the deployment of visual attention and how do they do it? Nature Reviews Neuroscience, 5, 495-501.

YASUDA, T. (2005). Effects of configural information on face shape perception. Japanese Journal of Psychology, 76, 131-138.

\section{NOTE}

1. We thank Kyle Cave and Martin Batty for contributing this insight.

(Manuscript received January 15, 2007; revision accepted for publication March 5, 2008.) 